\title{
THE CONJECTURE OF LANGLANDS AND RAPOPORT FOR SIEGEL MODULAR VARIETIES
}

\section{J. S. MILNE}

Frequently one attaches to an arithmetic object a zeta function whose analytic behavior reflects deep properties of the object. For example, attached to $\mathbf{Q}$ there is the Riemann zeta function, whose behavior (especially the position of its zeros) contains information about the distribution of the prime numbers. Dedekind attached a zeta function to every algebraic number field, and Hasse and Weil attached a zeta function to every algebraic variety over a number field. A central point of Langlands's philosophy is that these zeta functions should be among the $L$-functions that he attaches to automorphic representations of reductive groups.

A natural first testing ground for this idea is the family of Shimura varieties. For the simplest Shimura varieties, the elliptic modular curves, the results of Eichler and Shimura can be interpreted as demonstrating its validity. In general, to define a Shimura variety, one needs a reductive group $G$ over $\mathbf{Q}$ and a finite disjoint union $X$ of bounded symmetric domains on which $G(\mathbf{R})$ acts. For example, to define the elliptic modular curves, one takes $G$ to be $G L_{2}$ and $X$ to be the complex plane minus the real axis. The Shimura variety is then a family of varieties $\operatorname{Sh}(G, X)$ over $\mathbf{C}$, but a deep result says that it has a canonical model over a number field $E$. For almost all primes $v$ of $E$, this model reduces to a family of nonsingular varieties $\mathbf{S}_{v}$ over the finite residue field $k(v)$ at $v$, and the zeta function of the Shimura variety is defined in terms of the number of points of $S_{v}$ with coordinates in the finite fields containing $k(v)$.

Let $\mathbf{F}$ be the algebraic closure of $k(v)$. The starting point of Langlands's program for realizing the zeta function of $\operatorname{Sh}(G, X)$ as an automorphic $L$-function is to obtain an explicit description of the set of points on the Shimura variety with coordinates in $\mathbf{F}$, together with the action of the Frobenius automorphism of $\mathbf{F}$ and

Received by the editors September 20, 1990.

1980 Mathematics Subject Classification (1985 Revision). Primary 11G18.

Partially supported by the National Science Foundation. 
the Hecke operators on the set; the description should be directly in terms $G$ and $X$. Such a description was first conjectured in Langlands (1976), and the conjecture was made significantly more precise in Langlands and Rapoport (1987). Here we sketch a proof of this conjecture of Langlands and Rapoport in the key case of Siegel modular varieties. The same argument suffices to prove the conjecture for other Shimura varieties that are moduli varieties for polarized abelian varieties with endomorphism and level structures (at their primes of good reduction).

The philosophy underlying the conjecture of Langlands and Rapoport is that the Shimura variety should parameterize motives with additional structure over the field $\mathbf{F}$, and their conjecture is stated in terms of an object $\mathfrak{P}$, "the pseudo-motivic Galois group", which they predict describes the category of motives over F. To add plausibility to their conjecture, they showed that if one assumes Grothendieck's standard conjectures and the Tate conjecture for varieties over $\mathbf{F}$, and the Hodge conjecture for abelian varieties of $\mathbf{C M}$-type over $\mathbf{C}$, then it follows that $\mathfrak{P}$ does describe the category of motives over $\mathbf{F}$, and this then enabled them to deduce the validity of their conjecture for Siegel modular varieties. Thus the purpose of the present announcement is to indicate how the same result can be obtained without assumptions.

\section{The PSeudo-motivic Galois group}

(Langlands and Rapoport 1987, $\S \S 2$ and 3). Let $k$ be a field with algebraic closure $k^{\text {al }}$, and let $G$ be a pro-algebraic group over $k^{\text {al }}$. We shall need to consider extensions

$$
1 \rightarrow G\left(k^{\mathrm{al}}\right) \rightarrow \mathfrak{G} \rightarrow \mathrm{Gal}\left(k^{\mathrm{al}} / k\right) \rightarrow 1
$$

satisfying certain natural conditions. Two homomorphisms $\varphi$, $\varphi^{\prime}$ from one such extension $\mathfrak{G}$ to a second $\mathfrak{G}^{\prime}$ are said to be equivalent, denoted $\varphi \approx \varphi^{\prime}$, if there exists a $g \in G^{\prime}\left(k^{\mathrm{al}}\right)$ such that $\operatorname{ad}(g) \circ \varphi=\varphi^{\prime}$. From an extension $\mathfrak{G}$ over $\mathbf{Q}$, we obtain an extension $\mathfrak{G}(\ell)$ over $\mathbf{Q}_{\ell}$ by pulling back by $\operatorname{Gal}\left(\mathbf{Q}_{\ell}^{\text {al }} / \mathbf{Q}_{\ell}\right) \hookrightarrow$ $\operatorname{Gal}\left(\mathbf{Q}^{\mathrm{al}} / \mathbf{Q}\right)$ and pushing out by $G\left(\mathbf{Q}^{\mathrm{al}}\right) \hookrightarrow G\left(\mathbf{Q}_{\ell}^{\mathrm{al}}\right)$.

Define $\mathfrak{G}_{\infty}$ to be the Weil group of $\mathbf{R}$ regarded as an extension of $\mathrm{Gal}(\mathbf{C} / \mathbf{R})$ by $\mathbf{G}_{m}(\mathbf{C}), \mathfrak{G}_{\ell}$ to be the trivial extension $\{1\} \rtimes$ $\operatorname{Gal}\left(\mathbf{Q}_{\ell}^{\mathrm{al}} / \mathbf{Q}_{\ell}\right)$, and $\mathfrak{G}_{p}$ to be the Weil group of $\mathbf{Q}_{p}$ regarded as an extension of $\operatorname{Gal}\left(\mathbf{Q}_{p}^{\mathrm{al}} / \mathbf{Q}_{p}\right)$ by $\mathbf{G}\left(\mathbf{Q}_{p}^{\mathrm{al}}\right)$, where $\mathbf{G}$ is the universal covering group of $\mathbf{G}_{m}$. The pseudo-motivic Galois group is an 
extension

$$
1 \rightarrow P\left(\mathbf{Q}^{\mathrm{al}}\right) \rightarrow \mathfrak{P} \rightarrow \operatorname{Gal}\left(\mathbf{Q}^{\mathrm{al}} / \mathbf{Q}\right) \rightarrow 1
$$

with $P$ a pro-torus over $\mathbf{Q}$ whose character group is generated by Weil numbers. It is equipped with homomorphisms $\zeta_{\ell}: \mathfrak{G}_{\ell} \rightarrow$ $\mathfrak{P}(\ell)$ for all $\ell$ (including $p$ and $\infty)$, and the system $\left(\mathfrak{P},\left(\zeta_{\ell}\right)\right.$ ) is well defined up to a nonunique isomorphism.

\section{THE CONJECTURE}

Let $\operatorname{Sh}(G, X)$ be the Shimura variety defined by a reductive group $G$ over $\mathbf{Q}$ and a $G(\mathbf{R})$-conjugacy class $X$ of homomorphisms $\mathbf{C}^{\times} \rightarrow G(\mathbf{R})$ (see Deligne 1979, 2.1). It is known that $\operatorname{Sh}(G, X)$ has a canonical model $\operatorname{Sh}(G, X)_{E}$ over a certain number field $E(G, X)$ (the reflex field). Let $p$ be a prime for which there is a hyperspecial subgroup $K_{p}$ in $G\left(\mathbf{Q}_{p}\right)$, and choose a prime $v$ of $E(G, X)$ lying over $p$. It is conjectured that $\operatorname{Sh}_{p}(G, X)=_{d f}$ $\operatorname{Sh}(G, X) / K_{p}$ extends canonically to a smooth scheme over the ring of integers in $E(G, X)_{v}$, and so we can speak of the points $\mathrm{Sh}_{p}(\mathbf{F})$ of this scheme with coordinates in the algebraic closure $\mathbf{F}$ of $\mathbf{F}_{p}$. The Frobenius automorphism of $\mathbf{F}$ over $k(v)$ defines an automorphism $\Phi$ of $\operatorname{Sh}_{p}(\mathbf{F})$, and it is the pair $\left(\operatorname{Sh}_{p}(\mathbf{F}), \Phi\right)$, together with the action of the group $G\left(\mathbf{A}_{f}^{p}\right)$ of Hecke operators, that the conjecture of Langlands and Rapoport describes. Here $\mathbf{A}_{f}^{p}=\left(\prod_{\ell \neq p, \infty} \mathbf{Z}_{\ell}\right) \otimes \mathbf{Q}$.

Let $\mathfrak{G}_{G}$ be the split extension $G\left(\mathbf{Q}^{\mathrm{al}}\right) \rtimes \operatorname{Gal}\left(\mathbf{Q}^{\mathrm{al}} / \mathbf{Q}\right)$. Langlands and Rapoport define the notion of a homomorphism of extensions $\varphi: \mathfrak{P} \rightarrow \mathfrak{G}_{G}$ being admissible, and they attach to each admissible $\varphi$ a set with operator $(S(\varphi), \Phi(\varphi))$. Their conjecture then asserts that there is a $G\left(\mathbf{A}_{f}^{p}\right)$-equivariant isomorphism from $\left(\operatorname{Sh}_{p}(\mathbf{F}), \Phi\right)$ to the disjoint union of the sets $(S(\varphi), \Phi(\varphi))$ with $\varphi$ running over the equivalence classes of admissible $\varphi$.

\section{THE MODULI PROBLEM}

Fix a vector space $V$ over $\mathbf{Q}$ of dimension $2 \mathrm{~g}$ together with a nondegenerate skew-symmetric form $\psi$, and let $G$ be the corresponding group of symplectic similitudes and $X$ the corresponding Siegel double space (Deligne 1979, 1.3.1). The Shimura variety defined by $(G, X)$ and a compact open subgroup $K$ of $G\left(\mathbf{A}_{f}\right)$ parameterizes polarized abelian varieties over $\mathbf{C}$ with level structure. Results of Mumford show that this description remains valid over $\mathbf{Z}_{p}$ provided $p$ does not divide the level structure. Thus the 
conjecture in the first paragraph of $\S 2$ is true, and there is a modular description for $\operatorname{Sh}_{p}(\mathbf{F})$ which we now explain.

For any ring $R$, an $R$-isogeny $\mathrm{A} \rightarrow B$ of abelian varieties over $\mathrm{F}$ is an element of $\operatorname{Hom}(A, B) \otimes R$ having an inverse in $\operatorname{Hom}(B, A) \otimes R$. When $R \subset \mathbf{R}$, an $R$-polarization is an $R$ isogeny $\lambda: A \rightarrow A^{\vee}$ whose image in $\operatorname{Hom}\left(A, A^{\vee}\right) \otimes_{R} \mathbf{R}$ lies in the positive cone. Now $\mathrm{Sh}_{p}(\mathbf{F})$ can be identified with the set of $\mathbf{Z}_{(p)}$-isogeny classes of triples $(A, \lambda, \eta)$, where $\mathrm{A}$ is an abelian variety over $\mathbf{F}, \lambda$ is a $\mathbf{Z}_{(p)}$-polarization of $\mathrm{A}$, and $\eta$ is an isomorphism $V \otimes \mathbf{A}_{f}^{p} \rightarrow V_{f}^{p} A$ respecting the symplectic structures. Here $V_{f}^{p} A=\left(\prod_{\ell \neq p} T_{\ell} A\right) \otimes \mathbf{Q}$.

\section{Abelian varieties over $\mathbf{F}$}

Let $(A, \lambda)$ be a polarized abelian variety over $\mathbf{F}$ of dimension $g$. There is a representation $\rho$ of $\operatorname{End}(A) \otimes \mathbf{Q}$ on $V \otimes \mathbf{Q}^{\text {al }}$ such that

(a) for all $a \in \operatorname{End}(A)$, the characteristic polynomial of $\rho(a)$ on $V$ is the characteristic polynomial of a on $\mathrm{A}$;

(b) for all $x, y \in V \otimes \mathbf{Q}^{\text {al }}$ and $a \in \operatorname{End}(A) \otimes \mathbf{Q}, \psi(\rho(a) x, y)=$ $\psi\left(a, \rho\left(a^{*}\right) y\right)$, where $a \mapsto a^{*}$ is the Rosati involution on $\operatorname{End}(A) \otimes$ $\mathbf{Q}$ defined by $\lambda$.

From $\rho$, we can construct an extension

$$
1 \rightarrow Q_{A}\left(\mathbf{Q}^{\mathrm{al}}\right) \rightarrow \mathfrak{Q}(A, \lambda, \rho) \rightarrow \operatorname{Gal}\left(\mathbf{Q}^{\mathrm{al}} / \mathbf{Q}\right) \rightarrow 1
$$

by setting $\pi^{-1}(\tau)$ equal to the set of symplectic similitudes $(V, \psi) \otimes$ $\mathbf{Q}^{\text {al }} \rightarrow(V, \psi) \otimes \mathbf{Q}^{a l}$ intertwining ${ }^{\tau} \rho$ and $\rho$. The kernel $Q_{A}$ is a torus such that

$$
Q_{A}(\mathbf{Q})=\left\{a \in F^{\times} \mid a \cdot l a \in \mathbf{Q}^{\times}\right\}
$$

where $F$ is the centre of $\operatorname{End}(A) \otimes \mathbf{Q}$ and $l$ denotes complex conjugation. By construction, $\mathfrak{Q}(A, \lambda, \rho) \subset \mathfrak{G}_{G}$. From the various homology groups of A, we obtain homomorphisms $\zeta_{A, \ell}: \mathfrak{G}_{\ell} \rightarrow$ $\mathfrak{Q}(A, \lambda, \rho)$ for all $\ell$ (including $p$ and $\infty$ ).

\section{AN ISOGENY CLASS}

Fix a polarized abelian variety $\left(A_{0}, \lambda_{0}\right)$ over $\mathbf{F}$, and let $\operatorname{Sh}_{p}(\mathbf{F})_{0}$ be the subset of $\operatorname{Sh}_{p}(\mathbf{F})$ consisting of classes represented by triples $(A, \lambda, \eta)$ with $(A, \lambda)$ Q-isogenous to $\left(A_{0}, \lambda_{0}\right)$. The Frobenius endomorphism stabilizes $\operatorname{Sh}_{0}(\mathbf{F})$ and we write $\Phi_{0}$ for its restriction to $\mathrm{Sh}_{0}(\mathbf{F})$. Choose a representation $\rho$ as in $\S 4$, and let $\mathfrak{Q}=\mathfrak{Q}\left(A_{0}, \lambda_{0}, \rho\right)$. 
Proposition. Let $\varphi$ be a homomorphism $\mathfrak{P} \rightarrow \mathfrak{Q}$ such that $\varphi(\ell)$ 。 $\zeta_{\ell} \approx \zeta_{A_{0}, \ell}$ for all $\ell$; then there exists a $G\left(\mathbf{A}_{f}^{p}\right)$-equivariant bijection $(S(\varphi), \boldsymbol{\Phi}(\varphi)) \rightarrow\left(S h_{p}(\mathbf{F})_{0}, \Phi_{0}\right)$.

\section{A RESUlt OF LANGLANDS AND RAPOPORT}

Let $T$ be a torus over $\mathbf{Q}$, and let $\mu$ be a cocharacter of $T$ satisfying the following condition: ( $l=$ complex conjugation)

$$
(\tau-1)(l+1) \mu=0=(l+1)(\tau-1) \mu, \quad \text { all } \tau \in \operatorname{Gal}\left(\mathbf{Q}^{\text {al }} / \mathbf{Q}\right) .
$$

Let $\mathfrak{G}_{T}$ be the split extension $T\left(\mathbf{Q}^{\text {al }}\right) \rtimes \operatorname{Gal}\left(\mathbf{Q}^{\text {al }} / \mathbf{Q}\right)$. Then Langlands and Rapoport construct a homomorphism $\varphi_{\mu}: \mathfrak{P} \rightarrow \mathfrak{G}_{T}$, well defined up to equivalence (ibid. Satz 2.3).

Let $x$ be a special point of $X$. By definition this means that there is a maximal torus $T \subset G$ such that the cocharacter $\mu_{x}$ of $G_{\mathrm{C}}$ associated with $x$ factors through $T_{\mathbf{C}}$. When regarded as a cocharacter of $T, \mu_{x}$ satisfies the above condition, and the corresponding homomorphism $\varphi_{x}: \mathfrak{P} \rightarrow \mathfrak{G}_{T} \subset \mathfrak{G}_{G}$ is admissible; moreover, every admissible homomorphism is equivalent to one of this form (ibid. 5.2, 5.3).

\section{A RESULT OF KotTwITZ}

Let $E$ be a product of CM-fields, and let $(A, \lambda)$ be a polarized abelian variety over $\mathbf{Q}^{\text {al }}$ with complex multiplication by $E$. Let $T$ be the torus whose rational points are $\left\{a \in E^{\times} \mid a \cdot l a \in \mathbf{Q}^{\times}\right\}$. The action of $E$ on the tangent space to $A$ at 0 defines a CMtype $\Phi$ for $E$, and hence a cocharacter $\mu_{\Phi}$ of $T$ such that $\tau \circ \mu_{\Phi}$ is 1 or 0 according as $\tau \in \Phi$ or not. It satisfies the condition of $\S 6$, and so we obtain a homomorphism $\varphi_{\Phi}: \mathfrak{P} \rightarrow \mathfrak{G}_{T}$. By reduction modulo $p$, we obtain from $(A, \lambda)$ a similar pair $\left(A_{0}, \lambda_{0}\right)$ over $F$, still with complex multiplication by $E$. When we choose the representation $\rho$ of $\operatorname{End}\left(A_{0}\right) \otimes \mathbf{Q}$ in $\S 4$ so that $\rho(E)$ stabilizes $V$, then there will be a canonical embedding $i$ of $\mathfrak{Q}\left(A_{0}, \lambda_{0}, \rho\right)$ into $\mathfrak{G}_{T}$.

Proposition. For all $\ell$, including $p$ and $\infty, i \circ \zeta_{A_{0}, \ell} \approx \varphi_{\Phi}(\ell)$ (maps $\mathfrak{G}_{\ell} \rightarrow \mathfrak{G}_{T}(\ell)$ ).

Proof. The only difficult prime is $\ell=p$, and here the statement is equivalent to a conjecture of Kottwitz, which has been proved independently by him (unpublished, except for Kottwitz 1990, 12.1) and by Reimann and Zink (1988). 


\section{Associating AN ADMissible hoMomorphism With $\left(A_{0}, \lambda_{0}\right)$}

Let $\left(A_{0}, \lambda_{0}\right)$ be a polarized abelian variety over $\mathbf{F}$. We wish to define a homomorphism $\varphi_{0}: \mathfrak{P} \rightarrow \mathfrak{Q}\left(A_{0}, \lambda_{0}, \rho\right)$, for a suitable $\rho$, such that $\varphi_{0}(\ell) \circ \zeta_{\ell} \approx \zeta_{A_{0}, \ell}$ for all $\ell$.

On comparing the cohomology classes of $\mathfrak{P}$ and $\mathfrak{Q}$, one finds that there does exist a homomorphism $\varphi_{0}: \mathfrak{P} \rightarrow \mathfrak{Q}$. The obstruction to choosing $\varphi_{0}$ so that $\varphi_{0}(\ell) \circ \zeta_{\ell} \approx \zeta_{A_{0}, \ell}$ lies in $H^{1}\left(\mathbf{Q}, X_{*}\left(Q_{A_{0}}\right)\right.$ $\otimes C)$, where $C$ is the union of the idèle class groups of the subfields of $\mathbf{Q}^{a l}$. To prove that the obstruction is zero, realize $\left(A_{0}, \lambda_{0}\right)$ as in $\S 7$, and choose $\rho$ as in that section; then the existence of $\varphi_{\Phi}$ shows that the obstruction vanishes for $T$, and this implies that it vanishes for $Q_{A_{0}}$.

\section{COMPLETION OF THE PROOF}

Let $\mathscr{A}$ be the set of $\mathbf{Q}$-isogeny classes of polarized abelian varieties of dimension $g$ over $\mathbf{F}$, and let $\mathscr{M}$ be the set of equivalence classes of admissible homomorphisms $\mathfrak{P} \rightarrow \mathfrak{G}_{G}$. We have to find a bijection $[A, \lambda] \mapsto[\varphi]: \mathscr{A} \rightarrow \mathscr{M}$ such that $\varphi(\ell) \circ \zeta_{\ell} \approx \zeta_{A, \ell}$ for all $\ell$. Call two pairs $(A, \lambda)$ and $\left(A^{\prime}, \lambda^{\prime}\right)$ locally isogenous if $(A, \lambda)$ is $\mathbf{Q}_{\ell}$-isogenous to $\left(A^{\prime}, \lambda^{\prime}\right)$ for all $\ell$, and call two homomorphisms $\varphi$ and $\varphi^{\prime}$ locally equivalent if $\varphi(\ell) \circ \zeta_{\ell} \approx \varphi^{\prime}(\ell) \circ \zeta_{\ell}$ for all $\ell$. These relations define equivalence relations on $\mathscr{A}$ and $\mathscr{M}$, both of which we denote by $\sim$.

Proposition. There is a canonical bijection $\mathscr{A} / \sim \rightarrow \mathscr{M} / \sim$.

Proof. Given $(A, \lambda)$, choose $\varphi$ as in $\S 8$, and map the class of $(A, \lambda)$ to the class of $\varphi$. The surjectivity of the map follows from $\S \S 6$ and 7.

Now fix a pair $\left(A_{0}, \lambda_{0}\right)$, and choose a $\varphi_{0}$ as in $\S 8$. Write $\mathscr{A}\left(A_{0}, \lambda_{0}\right)$ for the equivalence class of $\left(A_{0}, \lambda_{0}\right)$ in $\mathscr{A} / \sim$ and $\mathscr{M}\left(A_{0}, \lambda_{0}\right)$ for the equivalence class of $\varphi_{0}$ in $\mathscr{M} / \sim$. It remains to show that there is a bijection $[A, \lambda] \mapsto[\varphi]: \mathscr{A}\left(A_{0}, \lambda_{0}\right) \rightarrow$ $\mathscr{M}\left(\varphi_{0}\right)$ such that $\varphi(\ell) \circ \zeta_{\ell} \approx \zeta_{A, \ell}$ for all $\ell$.

Let $I$ and $I^{\prime}$ be algebraic groups over $Q$ such that $I(Q)$ is the set of Q-isogenies $\left(A_{0}, \lambda_{0}\right) \rightarrow\left(A_{0}, \lambda_{0}\right)$ and $I^{\prime}(\mathbf{Q})=\{g \in$ $\left.G\left(\mathbf{Q}^{\mathrm{al}}\right) \mid \operatorname{ad}(g) \circ \varphi_{0}=\varphi_{0}\right\}$. There are canonical bijections $\mathscr{A}\left(A_{0}, \lambda_{0}\right)$ $\rightarrow \operatorname{Ker}^{1}(\mathbf{Q}, I)$ and $\mathscr{M}\left(\varphi_{0}\right) \rightarrow \operatorname{Ker}^{1}\left(\mathbf{Q}, I^{\prime}\right)$, where

$$
\operatorname{Ker}^{1}(\mathbf{Q}, *)=\operatorname{Ker}\left(H^{1}(\mathbf{Q}, *) \rightarrow \prod_{\text {all } \ell} H^{1}\left(\mathbf{Q}_{\ell}, *\right)\right) .
$$


Since $\rho$ defines an isomorphism $I \rightarrow I^{\prime}$, we see that there is a unique bijection $\mathscr{A}\left(A_{0}, \lambda_{0}\right) \rightarrow \mathscr{M}\left(\varphi_{0}\right)$ sending $\left(A_{0}, \lambda_{0}\right)$ to $\varphi_{0}$ and compatible with the bijection $\operatorname{Ker}^{1}(\mathbf{Q}, I) \rightarrow \operatorname{Ker}^{1}\left(\mathbf{Q}, I^{\prime}\right)$. This is the required map.

\section{KOTTWITZ's FORMULA}

Kottwitz has given a conjectural formula for the number of points mod $p$ on a Shimura variety with coordinates in a finite field as an explicit sum of (twisted) orbital integrals (Kottwitz $1990, \S 3$ ). This formula is implied by the conjecture of Langlands and Rapoport, but for Siegel modular varieties Kottwitz has obtained a proof of the formula that by-passes the conjecture (sketched, ibid. §12).

\section{REFERENCES}

P. Deligne, Variétés de Shimura: Interprétation modulaire, et techniques de construction de modèles canoniques, Proc. Sympos. Pure Math., vol. 33, Amer. Math. Soc., Providence, RI, 1979, pp. 247-290.

$R$. Kottwitz, Shimura varieties and $\lambda$-adic representations, Automorphic Forms, Shimura Varieties, and $L$-functions, Proceedings of a Conference held at the University of Michigan, Ann Arbor, July 6-16, 1988 (L. Clozel and J. S. Milne, eds.), Perspectives in Math., no. 10, Academic Press, Boston, 1990, pp. 161-209.

R. Langlands, Some contemporary problems with origin in the Jugendtraum, Mathematical Developments Arising from Hilbert's Problems, Amer. Math. Soc., Providence, RI, 1976, pp. 401-418.

R. Langlands and M. Rapoport, Shimuravarietäten und Gerben, J. Reine Angew. Math. 378 (1987), 113-220.

H. Reimann, and T. Zink, Der Dieudonnémodul einer polarisierten abelschen Mannigfaltigkeit vom CM-Typ, Ann. of Math. 128 (1988), 461-482.

Department of Mathematics, University of Michigan, ANn ARbor, Michigan 48109

E-mail address: JMilne@UB.cc.Umich.edu 
